\title{
Problématique pour une géographie «sociale» des espaces sociaux en Suisse
}

Dans le double cadre de la préparation de ce numéro spécial de GEOGRAPHICA HELVETICA consacré à la géographie sociale et de la préparation d'un vaste ouvrage collectif, Nouvelle géographie de la Suisse à paraître aux éditions Payot sous la responsabilité de Claude Raffestin et de l'auteur en 1987, nous nous sommes interrogés sur les tenants et aboutissants d'une problématique possible pour une géographie «sociale» des espaces sociaux en Suisse. Une problématique qui soit l'occasion d'un renouvellement critique et de la théorie sociale et de la société ellemême en même temps que celle d'une saisie nouvelle des principales dimensions des connaissances et des pratiques que la société suisse a de son espace.

Une telle ambition réclamait que l'on élucide d'abord le contenu d'une distinction indispensable: la géographie «sociale» est-elle l'équivalent d'une géographie des «espaces sociaux»? Si non, comment cette géographie des espaces sociaux s'inscritelle dans la variété des domaines possibles de la géographie sociale? Ce qui réclame qu'on s'interroge également sur diverses manières de la pratiquer. Ce n'est qu'à travers l'explicitation des réponses possibles à ces trois questions qu'à la lumière de notre saisie des spécificités suisses, et des pratiques et connaissances que nous pouvons en avoir, à travers les données disponibles certes, mais aussi des connaissances que cette société a déjà produites ou suscitées, nous pourrons situer le profil et l'esprit de notre propre contribution dans le livre qui s'annonce.

\section{Géographie sociale et géographie des espaces sociaux}

A la question: "géographie sociale et géographie des espaces sociaux sont-elles équivalentes?» la réponse est évidemment négative.

D'abord parce que la première renvoie à une spécification du domaine et du point de vue particulier de notre discipline parmi les différentes manières de comprendre le monde géographiquement, parmi les différents points de vue et domaines pouvant être les siens. Ce point de vue, c'est le point de vue social de l'homme, à travers l'étude d'un domaine précis, celui des rapports que les hommes entretiennent en- tre eux, et qui peuvent être qualifiées de rapports sociaux. Comme tels, ils constituent le champ d'étude privilégié de la sociologie mais il peuvent être qualifiés de rapports géographiques dans la mesure où, en termes de production, d'habitat, de loisirs, de déplacements... ils unissent les hommes aux lieux, à l'espace, à travers une grande variété de rapports encore, «rapports économiques de production», mais aussi «rapports écologiques (d'adaptation au milieu naturel), historiques (de permanence plus ou moins longue, psychologiques (d'adaptation et d'attachement, ou d'inadaptation et de répulsion), juridiques (règles d'usage, de transmission, d'exploitation de la propriété par exemple) ou encore idéologiques (définissant la fonction idéologique de certains lieux)»(FRÉMONT, 1984). L'objet de la géographie sociale est bien, fondamentalement, l'étude des rapports existant entre rapports sociaux et rapports géographiques et des combinaisons (spatiales) qui en dérivent, à toutes les échelles, du local à l'international, selon lesquelles «ces rapports de rapports» (FRÉMONT) s'emboîtent, interfèrent et évoluent. Ils définissent ainsi une grande variété d'espaces sociaux, "toujours mutants mais relativement stables», des «espaces-temps» territoriaux en fait (BAREL, 1986), qu'organisent les diverses activités économiques et socio-culturelles des hommes et les rapports qu'ils entretiennent entre eux et avec elles. Ces rapports impliquent nécessairement une non moins grande variété de médiations possibles dont trois sans doute paraissent dominantes même à qui veut éviter de tomber dans le déterminisme idéologique d'une quelconque «instance»: la division sociale du travail d'une part - et, corrélativement, l'ensemble des rapports passant par ou s'inscrivant dans la mobilité spatiale -, les ségrégations de toutes sortes, liées aux "concurrences très fortes en un lieu - ou milieu - saturé, raréfié, convoité» (AURIAC, 1986).

Mais la géographie des «espaces sociaux» en tant que tels, parce que se voulant globale, et pas simplement géographie des «questions sociales» ou sociologie des faits spatiaux, n'est pas que «sociale»: elle

Jean-Bernard Racine, Prof., Dr, Institut de Géographie de I'Université de Lausanne, Dorigny, 1015 Lausanne. 
s'attache certes à l'une des dimensions possibles de la géographicité du monde (DARDEL, 1952), celle qui nait de la convergence, dans le réel social, des articulations spatiales et sociales du monde vécu. Mais ce faisant elle s'appuie sur la connaissance et la saisie de l'ensemble des rapports unissant les faits sociaux à l'espace, qu'ils relèvent de l'économique, du politique et de l'idéologique. Nous admettrons en effet, d'entrée de jeu et quasi axiomatiquement, avec R. FOSSAERT (1977) et dans la ligne des enseignements de MARX les plus marqués du sceau de l'évidence, «que toute société doit (nécessairement) produire et reproduire ses conditions matérielles d'existence; toute société doit organiser, d'une manière ou d'une autre, la collectivité humaine qui l'habite; toute société doit (ou plus exactement ne peut pas ne pas) se former une représentation du monde où elle vit... les hommes (vivants) dans une même société (étant) toujours pris, à des titres et à des degrés divers, dans des relations qui participent des trois niveaux». Une géographie sociale («le groupe humain d'abord, l'espace ensuite» plaidait Renée ROCHEFORT dans les années 60), cherchera donc dans quelle mesure ces relations se structurent d'une manière identifiable dans le cadre d'espaces «socio-géographiques» que l'on puisse circonscrire sur une carte, ne serait-ce que par des contours séparant à grands traits ce qui relève d'une certaine réalité, d'une certaine appartenance, de certaines solidarités, de ce qui n'en relève plus, ou relève de ce qui est autour.

Nous postulons ainsi que l'espace se divise, à travers l'interaction locale des facteurs, en sous-systèmes reconnaissables de relations sociales plus ou moins structurées, irréductibles à leurs voisins, auxquels ils ressemblent par certains points, auxquels ils s'opposent radicalement par d'autres (BRUNET, 1986). Ce qui revient à chercher, en d'autres termes, des sous-ensembles qui soient tels que la variance interne tende à être minimale en leur sein alors qu'elle tendra à être maximale quand on passe d'un sousensemble à un autre (RACINE et REYMOND, 1973).

Mais le réel social est fondamentalement un réel pratiqué, c'est-à-dire vécu, chacune des relations vécues pouvant définir de nouveaux espaces, espaces d'action et d'interconnaissance auxquels correspond mieux l'idée d'espace social (CLAVAL, 1973). C'est tout ce qui est impliqué par ces pratiques que les géographies «dites» de la perception, des comportements et des représentations (BAILLY, 1984) tentent de saisir quand elles abordent l'espace «par le vécu» et plus particulièrement ces «lieux privilégiés de manifestation des diverses formes de sociabilité» qu'elles cherchent à identifier pour en analyser les fonctions et les significations possibles (RACINE, 1985) - quand du moins elles veulent bien ne pas en rester, au pire, à une simple géographie des «impressions» et des états d'âme, géographie onirique susceptible de remplir une sorte de musée imagi- naire, «anthropologie paresseuse qui a toujours rendu compte de la perception comme d'un simple enregistrement d'une donnée, d'un désir, comme d'un phénomène d'attraction par l'objet absent ou distant» (DEMATTEIS, 1985), au mieux, et plus légitimement, à une géographie des mentalités.

Reste donc à savoir si les analyses décrivant l'espace social, de nos agglomérations par exemple, tel qu'on peut certes en saisir certains contenus et contours par des séries statistiques et leurs combinaisons particulières, rendront jamais compte de l'espace social tel qu'il est connu (et richement exprimé), dans la qualité vécu du quotidien, par un Fritz ZORN (1979) par exemple nous révélant, «dans le meilleur des mondes possibles», le monde "passablement dégénéré» (p. 29) de la Rive dorée du lac de Zurich, «univers hostile à la vie, système d'où étaient exclus tous les rapports, tous les conflits, les choses du monde glissant en se croisant sans la moindre friction» (p. 43), monde menteur (p. 31) où «le masque de sérénité du spectateur de la vie s'obtient au prix de la castration de l'âme (p. 200), monde dans lequel l'auteur de Mars se dit assassiné, dévoré par ses parents, co-victimes (p. 49) des valeurs de la société qu'ils incarnent, société dont les enfants meurent de l'incarner parfaitement. Monde «criminel», mais périssable, monde contesté aussi, comme on le sait, même en Suisse et par des Suisses.

A cet égard, la question se pose aussi: nos morphologies et autres stratigraphies sociales, ne survolentelles pas souvent, sans les voir, parce qu'uniquement saisissables à «meilleure» échelle, ces «espaces de l'ombre» d'un Monde qui ne fonctionnerait pas sans l'Antimonde qu'il secrète en permanence et qu'évoque Roger BRUNET (1986)? Antimonde des espaces spécialisés, en forme de zones d'ombre, dont certaines sont fort bien structurées, voire permanentes. "Arrières-cuisines» de la reproduction économique et sociale d'un pays rigide et moralisateur, espaces de la drogue et des trafics occultes, espaces du sexe commercialisé, espace des laissés pour compte de la scolarité, des exilés, des immigrés, des chômeurs, et, dans un autre contexte sans doute, espace des femmes qui, on commence à s'en rendre compte, a ses spécificitées, espaces de «l'informel» et du «noir», accueillant soit des activités tolérées et limtées, «tolérées parce que limitées», ou «cachant la population des déviants, des marginaux, des différents», espaces de la révolte parfois, quand soudain Zurich brûle et Lausanne bouge et expriment dans la rue et sur les murs, le refus brutal et primitif, par une "certaine jeunesse», comme on dit, d'une société étouffante et aliénée, règne de la solitude et de l'ennui, de l'absence de communication. Le besoin exprimé est finalement assez clair: besoin d'un espace de liberté, d'un territoire rêvé («Raser les Alpes pour qu'on voie la mer»), dont la pierre d'angle, le «centre autonome», est censé 
alors ménager à un jeune, et pour un temps, cet espace «laboratoire social», ce relais entre le milieu familial qu'il refuse et un champ social où il n'est pas encore inscrit.

Ces espaces, il faudrait bien les saisir aussi, dans leur originalité et leur fragilité. Ne sait-on pas déjà, cinq ans après, que les essais d'autogestion, les nouvelles coopératives, les entreprises alternatives, les foyers féministes, les nouveaux groupes artistiques... c'est ailleurs qu'à Zurich qu'il faudra désormais les chercher? «Zurich, fini le flip, retour au fric» titrait, dès 1982, un article de Domaine public recensant la parution de La vie...vite - Lausanne bouge, 1980-1981: une chronique (MÉNÉTREY et Collectif de défense, 1982).

Si la géographie sociale, comme «étude des configurations spatiales des rapports sociaux renvoie bien à une théorie des sociétés et des sociabilités par la recherche géographique de terrain confrontée aux changements sociauX» (HÉRIN, 1986), la notion d'espace vécu renvoie plus directement au monde de l'existence et donc de l'expérience humaine, en termes d'intention, de conséquences et de significations. La connotation donnée par HUSSERL au concept de Lebenswelt dépasse l'espace pour incorporer tous les faits vécus (POCOCK, 1984), mais les géographes l'ont assez bien saisie aujourd'hui (RACINE, 1985) pour renouveler leurs études des espaces sociaux, pour en faire une géographie de l'homme total et des types d'hommes (de femmes, d'enfants), dans leur expérience de la géographicité vécue, capable de saisir, à côté des éléments territoriaux matériels (techniques aussi bien que physiques et biologiques), d'autres éléments, immatériels ou symboliques, voire tout à la fois matériels et immatériels (la langue par exemple), jusqu'à comprendre, avec Yves BAREL, que «tout élément, même le plus physique ou biologique, n'entre dans la composition d'un territoire qu'après être passé par le crible d'un processus de symbolisation qui le "dématérialise" en quelque sorte», tant et si bien que «tout territoire social est un produit de l'imaginaire humain», tout se passant «comme si, prêtant sa voix aux territoires qu'il crée, l'imaginaire humain leur faisait dire: j'existe parce que j'ai du sens et j'ai du sens parce que j'existe» (BAREL, 1986).

Ces espaces vécus de l'expérience, de la mémorisation et de la valorisation individuelle et collective, subjective et intersubjective, cet espace signifiant jouant le rôle que l'on sait aujourd'hui dans l'identification humaine, forment sans doute les territoires d'une géographie sociale possible, allant bien audelà de la simple résurgence, actuellement à la mode en France (FRÉMONT, CHEVALIER, HÉRIN, RENARD. 1984), d'une géographie soit entièrement centrée sur le «moi», et ses discours, soit encore exclusivement occupée à décrire et à expliquer, par MARX, les conditions d'existence des catégories défavori- sées, ouvriers, chômeurs, marginaux, laissés pour compte du système scolaire, personnes âgées, les femmes, cherchant ainsi à mettre les outils de la connaissance au service des opprimés pour les aider à résister aux mécanismes cachés qui régissent les rapports de force sur l'espace (HÉRIN, 1986), en bref, une géographie des «classes dominées». Mais, justement, ces orientations diverses obligent à reconnaître ici que le discours fondateur d'une pratique plus riche de notre discipline s'enracine, pour les uns comme pour les autres, dans une vision épistémique qu'il conviendrait, en chaque cas, d'expliciter. Quelques exemples suffiront à illustrer ce principe à notre avis essentiel.

\section{De la morphologie sociale à l'étude des territorialités}

Conçu dans son sens le plus large, une géographie sociale de la Suisse s'obligera sans doute à étudier, à divers niveaux d'agrégation des données, une grande variété de domaines. Dans un premier temps il s'agira bien d'une simple «application de la méthode géographique aux données sociales», à l'image de la géographie sociale que nous proposait P. GEORGE autour des années 40 en France, ces données (sociales) étant simplement domiciliées dans l'espace par la représentation cartographique permettant de faire figurer chaque fait ou combinaison particulière de faits à leur échelle et à leur place exacte. Le travail consisterait, pour l'essentiel, en un effort de classement, fondé sur la description et la localisation des différents types de structures sociales au sein du territoire helvétique, en essayant, si possible, de saisir le sens des modifications en cours. Contribution partielle où le social en fait ne correspondrait encore ni à une variable dépendante, à expliquer, ni à la variable indépendante, explicative, puisqu'il ne s'agirait que de contribuer à la mise en place d'un élément, parmi d'autres, de la différenciation globale du pays. Telle carte présenterait, à une échelle particulière, les variations spatiales des conditions d'insertion de l'emploi de la force de travail potentielle, telle autre celle du sous-emploi, telle autre enfin celle des revenus moyens par ménage.

Aller plus loin, dans l'élaboration d'une véritable géographie du travail par exemple (GEORGE, 1978, RAFFESTIN et BRESSO, 1979), réclame déjà un important travail de préparation des données, dans un double effort de reconstruction théorique (et graphique) de la structure sociale, préparant le passage de la notion de catégorie socio-professionnelle aux notions de classes et de structures sociales, comme un travail de C. LALIVE d'EPINAY, E.CHRISTIE et M. BASSAND (1978) en a donné l'image, et de saisie conjoncturelle des conditions des techniques et de l'organisation économique et sociale dans lesquelles 
s'inscrit l'emploi et le sous-emploi de la force de travail préalablement qualifiée. Ce type de description des configurations spatiales du social peut (et doit sans doute) se prolonger par l'étude des mécanismes de production et des relations socio-spatiales qui en dépendent, telles que médiatisées entre autres par les rapports de propriété. Rien n'interdit alors d'évoquer un certain nombre d'articulations que l'on pourrait considérer comme résidus des différents modes de production au sein du mode de production dominant, lui-même morphologie «locale», «appartenant à et en interaction avec» une morphologie "globale» dénotée "formation économique et sociale helvétique». Mais il s'agirait surtout de chercher les acteurs commandant l'ensemble des médiations (rôle des bourgeoisies locales, des propriétaires des moyens de production, qu'il s'agisse de bien fonciers, ruraux, de biens industriels ou commerciaux, que leur origine et leur inscription soit purement locale ou nationale, voire internationale). Mais ne déborderait-on pas déjà, à ce niveau, les limites d'une géographie sociale, en s'épuisant peut-être dans l'explicitation de ses déterminations économiques? Les «architectures sociales», comme Paul Claval l'a bien montré (1973) sont à l'évidence plus complexes que celles dérivant des seuls rapports de production, comme le sont aussi, «la manière de les contrôler, de les infléchir, de les manipuler».

Déjà, la généralisation des méthodes d'analyse multivariée qui, dans les années 60 et 70 , ont renouvelé l'inspiration «écologique» des géographes anglosaxons, a permis de saisir comment une grande variété d'attributs sociaux de l'espace, généralement tirés des données de recensement qualifiant les populations en leur lieu de résidence, au plan démographique (âge et sexe), «social» (éducation, revenus, emplois), culturel (langue et religion), son liés entre eux étant donné les éléments (les secteurs de recensement) qui les fixent et de quelle manière (par le biais des algorithmes taxinomiques) les éléments sont liés entre eux étant donné la combinaison particulière des attributs qu'ils portent - (RACINE et REYMOND, 1973). Ce faisant, le géographe transforme ses unités statistiques quantitatives de départ, en unités géographiques qualitatives. Il découvre des types de lieux structurés selon l'interdépendance complexe de leurs caractéristiques, telles qu'elles se combinent différemment de lieu à lieu, sans que ce travail représente une véritable rupture par rapport aux premiers travaux de l'école de P. GEORGE, sinon qu'il s'appuie, «systématiquement», sur les ressources de l'ordinateur.

Mais s'agit-il pour autant de la définition d'un espace social? Celui que l'on découvre ainsi n'est rien d'autre en fait que l'espace des catégories retenues dans les recensements officiels et les publications des agences gouvernementales, c'est-à-dire, finale- ment, un «social» construit par le pouvoir, ne décrivant que des co-occurrences de caractères sociaux dans des espaces politico-administratifs. On commence d'ailleurs à bien connaître aujourd'hui la manière dont se structurent, sur l'ensemble des communes suisses, des districts, des régions de mobilité spatiale, des cantons et des bassins d'emploi, les différentes variables indexant l'essentiel des caractéristiques démographiques, sociales, économiques et culturelles de la population helvétique. Toutes les études confirment l'importance primordiale d'une dimension centre-périphérie, indépendante des caractéristiques socio-culturelles (à l'exception des «étrangers» et des «sans religion»), cette première dimension étant orthogonalement recoupée par l'opposition sectorielle entre zones dominées par les activités secondaires et celles dominées par les hommes du tertiaire, d'autres composantes indépendantes «moins importantes», permettant de définir d'autres clivages encore, d'ordre socio-culturel et socio-économique, «moins importants» par les grappes de corrélatifs qui s'y associent de façon spécifique. Jouant à toutes les échelles de référence, la composante centre-périphérie indexe bien évidemment un profil, ou une échelle, d'urbanité, saisissable aussi bien entre cantons qu'au sein des agglomérations et autres zones urbaines. Les différents travaux publiés par l'IREC et ses équipes, avec ou sans l'aide de l'Institut de Géographie de l'Université de Zurich, et souvent dans le cadre du Programme national de recherches sur les "Problèmes régionaux», en témoignent à l'évidence (SCHULER et NEF, 1983). Nous les avons prolongés à l'Institut de Géographie de l'Université de Lausanne en donnant une couleur plus spécifiquement "sociale» au choix des attributs et en focalisant l'étude sur les communes «agglomérées», saisies soit dans leur ensemble, (218), soit telles que rattachées à chacune des cinq grandes métropoles du pays. Les résultats sont analogues. Cinq composantes regroupent l'essentiel de la variance contenue dans la matrice des données, dans l'ordre:

- le passage du rural à l'urbain (centre-périphérie) (avec leurs corrélatifs respectifs: actifs primaires, propriétaires, grands ménages, nombre d'habitants par logement, habitat unifamilial d'un côté; importance de la population, densité, logements multifamiliaux, ménages à une ou deux personnes, femmes actives de l'autre (cf. fig. 1).

- la spécialisation socio-professionnelle des communes urbaines, soit à dominante tertiaire (avec les hauts niveaux de scolarité atteints par leur population, le poids des employés supérieurs, le coût élevé des loyers, l'importance des hauts revenus, mais aussi l'importance des populations déclarant parler un "autre langage» et être «sans religion»), soit à dominante secondaire, avec leurs étrangers, italophones tout particulièrement, 
- le statut démographique et son habitat (les jeunes, les ménages à trois ou quatre personnes, les émigrants quotidiens parmi les actifs d'un côté, dans des communes dont le bâti créé entre 1976 et 1980 est important, par opposition aux communes peuplées de gens plus âgés, au bâti plus ancien, avec de tous petits ménages et beaucoup de bas revenus),

- la spécificité ethno-culturelle des communes d'emploi (avec leurs «autres religions» et «autres langues» encore, mais aussi une part importante d'étrangers et d'immigrants quotidiens par rapport aux emplois dans leur population),

- l'échelle du statut socio-économique enfin, combinant le jeu simultané des hauts revenus, des emplois supérieurs et des hauts loyers, facteur de ségrégation particulière soulignant, en dehors de l'existence d'une configuration spatiale indépendante de celle déjà définie par la spécialisation socio-professionnelle, des tâches d'aisance soupoudrant le pays, de Cologny et Collonge-Bellerive dans l'agglomération de Genève à Uitikon ou Zollikon et Zumikon dans celle de Zurich, les «rives dorées» des deux principaux centres helvétiques se faisant écho par dessus les luxueuses banlieues bâloises ou bernoises, celles de Lausanne restant à des «écarts» plus modestes par rapport à la moyenne des «scores», les hauts revenus de StSulpice ou Jouxtens-Mézery n'allant pas forcément de pair avec les loyers comparables à ceux qu'occupe la haute société zurichoise, genevoise ou bâloise (cf. fig. 2).

Dans leur étonnante stabilité transcalaire, tous ces résultats semblent bien confirmer la mise en place d'une structuration socio-spatiale originale d'un bout à l'autre du pays autour de ses centres principaux. On peut les prolonger aujourd'hui par la saisie de nouveaux indicateurs sociaux, axés sur la mesure de la qualité de la vie (RACINE, 1986) et du bien-être social, voire les compléter par (ou les confronter à) la saisie corrélative d'un ensemble de caractéristiques vécues, perçues ou exprimées par les habitants (ne serait-ce qu'à l'occasion des élections et des consultations électorales), mais aussi à travers la multiplication des sondages d'opinion ou, comme pour E. WALTER-BUSCH (1983), l'interrogation des jeunes recrues. Leur exploitation sera d'autant plus riche que l'on pourra confronter des analyses s'inscrivant non seulement dans une variété d'échelles, mais saisissant des ensembles spatiaux plus spécifiquement délimités, les modulations intervenant dans le jeu des variables pouvant être géographiquement significatives. Reste à savoir cependant si ces structures et combinaisons diverses qu'on en dérive spatialement correspondent réellement à une mosaïque de zones ou d'aires dont on puisse dire qu'elles ont une quelconque homogénéité en termes d'espaces perçus et vécus par leurs habitants, étant produits et appro- priés dans une praxis commune, appropriation dont ils auraient quelque conscience.

Toutes les études disponibles semblent bien nous confirmer qu'en dépit du caractère apparent d'identité sociale que prennent certains quartiers définis a priori, en dépit aussi de la possibilité de relier les principaux clivages sociaux et les types de sociabilité, à l'influence de certains types d'organisation sociale de l'espace (CAMPICHE et ZIMMERMANN, I. E. S., 1975), tels que vécus et pratiqués par des populations finalement irréductibles les unes aux autres, les espaces sociaux sont d'une telle variété, au sein même de quartiers dotés d'une «existence historique» incontestable, que force est de constater «la disparition des quartiers en tant que centres de vie sociale organique».

Les auteurs du travail sur les Eaux-Vives à Genève (I. E. S. 1975) insistent sur ce point: d'une manière générale, les personnes résidant depuis longtemps dans le quartier n'ont pas développé des relations de voisinage propres à les enraciner davantage que les autres dans l'unité sociale étudiée. Et pourtant, l'analyse factorielle de l'ensemble des caractéristiques recueillies sur les "profils» de la populations, socio-démographique, socio-économique, socioculturel et socio-religieux, accompagnées de données sur la mobilité géographique et la mobilité sociale, avait mis en évidence, comme première et principale composante, l'importance primordiale du clivage séparant les personnes dont le lieu de première socialisation fut Genève, de celles qui ont passé leur enfance ailleurs. Il n'y a donc pas forcément adéquation entre ce que définit ce facteur dit "d'intégration locale» au vu des attributs de la population, et les comportements réels tels qu'ils peuvent être décrits par la saisie, par questionnaires, des différents types de relations qu'entretiennent les habitants d'un quartier avec l'altérité, qu'il s'agisse, comme ici, de la proche famille, da la parenté, des voisins, du quartier, des amis, du travail, du syndicat, du parti politique, des sociétés et groupements, de la paroisse, des groupements religieux, des vacances, du militaire, autant de thèmes possibles d'une étude des "pratiques» sociales.

Conforté par d'autres résultats analogues, des deux côtés de l'océan Atlantique, cet énoncé conduit à mettre en cause la pertinence qu'il y a à vouloir considérer comme un tout, à la manière de l'écologie humaine, l'ensemble humain lui-même, dont l'habitat nous sert à définir le cadre spatial. On peut habiter le quartier des Eaux-Vives, et vivre, comme on dit, dans un «autre monde». Vivre à Genève sans jamais s'y recontrer. Ce n'est donc pas, en soi, un espace social, et ce en dépit du fait que dans le cadre spatial comme dans l'espace social censé a priori lui correspondre, certains points restent privilégiés, "points d'attraction qui constituent une structure dans la mesure où ils sont interdépendants dans une 

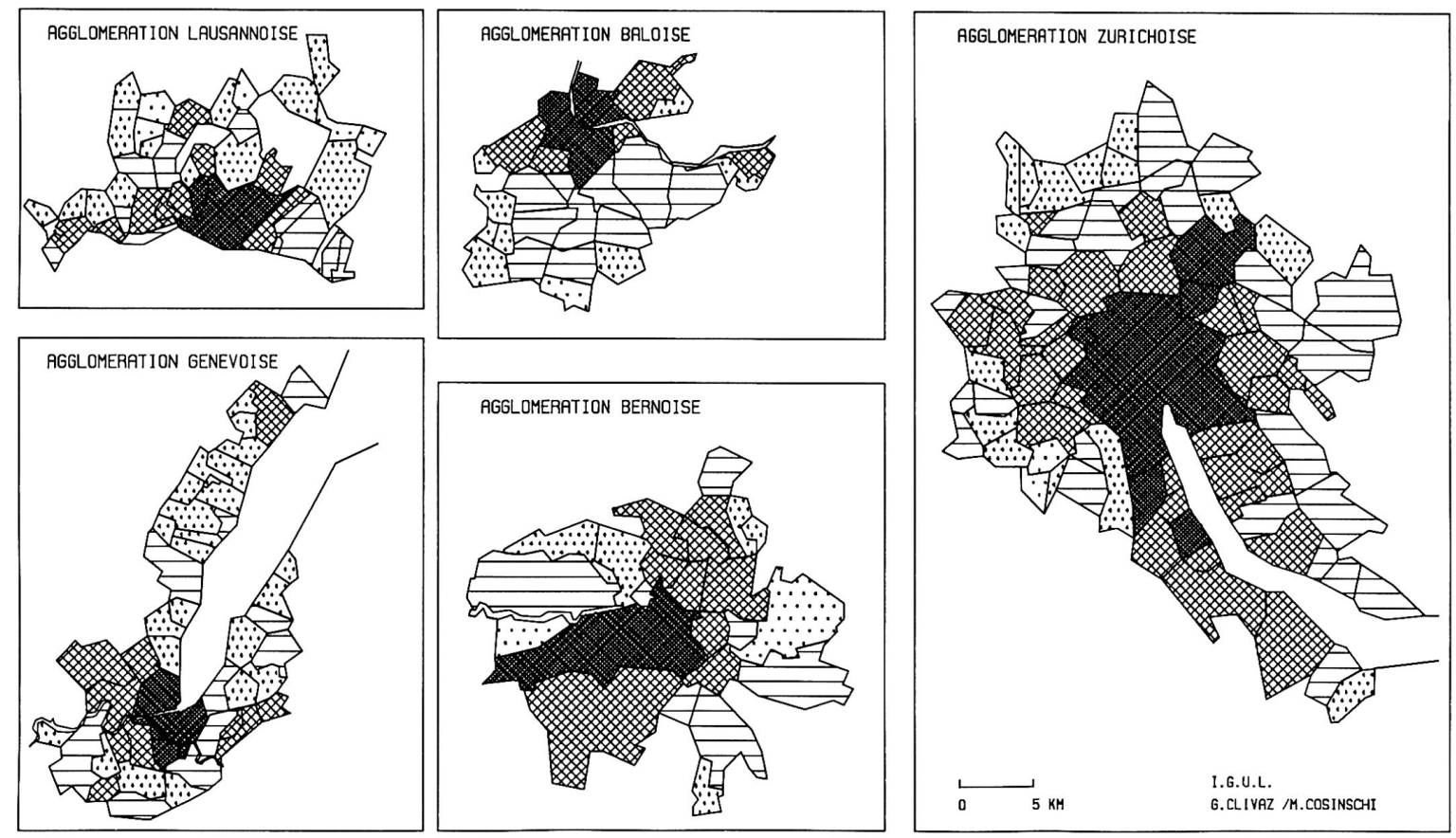

COMPOSANTE 1

DE LA CENTRALI.

TE URBAINE (ET

SES CORRELATIFS

A LA PERIPHE-

RIE URBAINE

$\nabla$

$20-3.0--1.50$

$-1.50--0.500$

$-0.500-0.500$

$0.500-1.50$

$\therefore \quad 1.50-3.0$

Figure 1 
ANALYSE STRUCTURELLE DE L'ESPACE SOCIAL DES AGGLOMERATIONS SUISSES
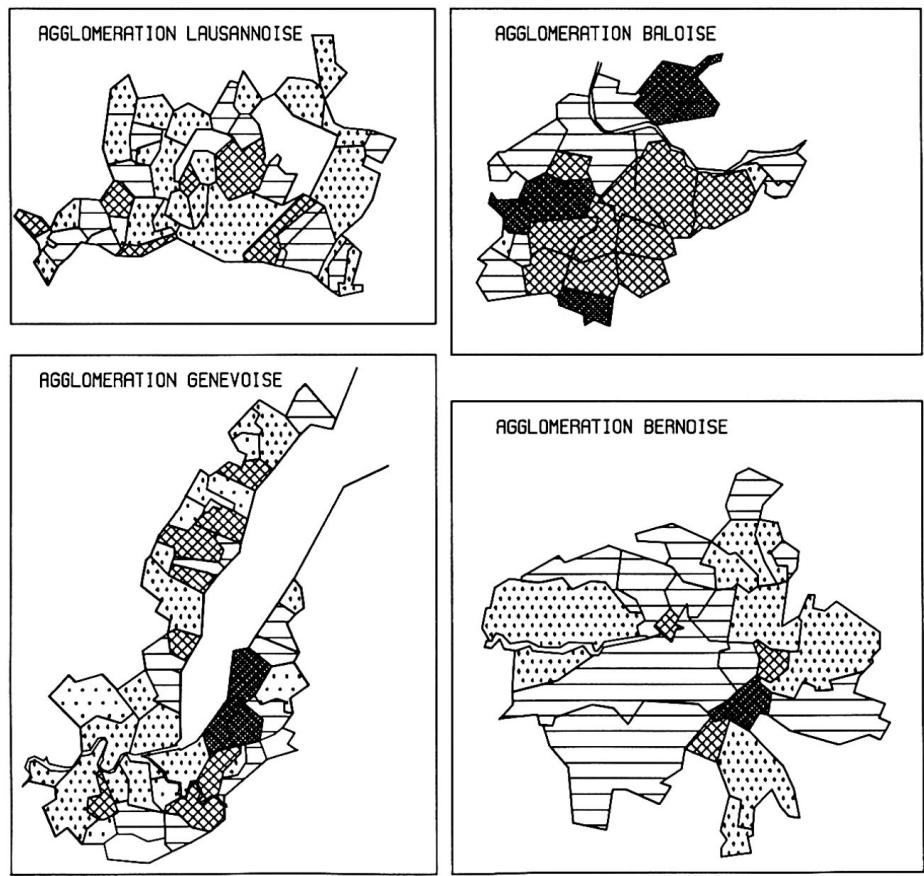

AGGLOMERATION BERNOISE

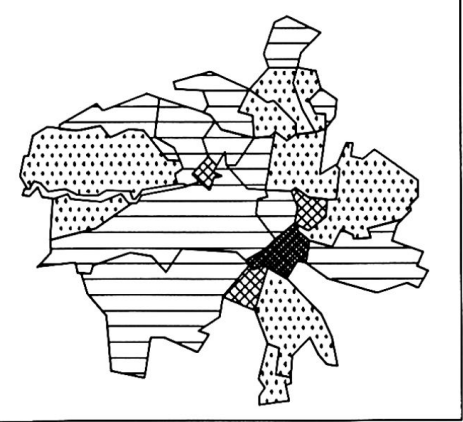

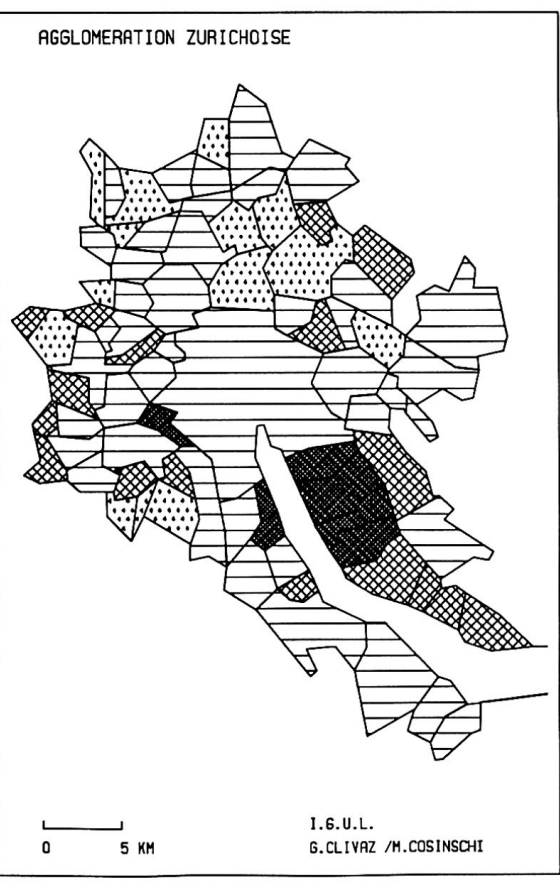

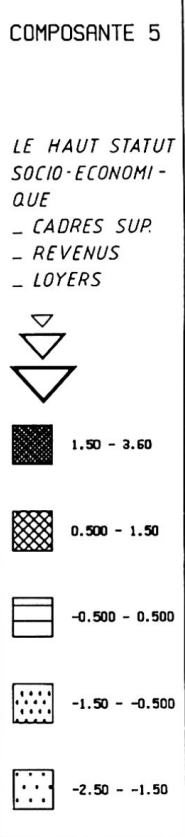

Figure 2 
totalité et où ils sont disposés dans un certain ordre, cet ordre correspondant par ailleurs à certains modèles culturels» (CAMPICHE et ZIMMERANN, 1975b).

On peut pousser la réflexion encore plus loin en se demandant si un regroupement, très généralement observé, comme celui des italiens - ou des revenus d'ailleurs -, crée vraiment un «espace social». En fait il est peut-être formé par lui, il le manifeste, le cristallise, étant sans doute d'autant plus attirant qu'il est structuré, le regroupement s'effectuant de fait parce qu'il existe, entre eux, un espace qu'il faut bien baptiser «social», espace où l'on s'agrège, dirait Roger Brunet (1986), par choix, par mimétisme, «autant et plus qu'on se ségrège, par répulsion, et d'un même mouvement souvent». C'est l'occasion de rappeler, avec le directeur de la Maison de la géographie à Montpellier, que les mêmes fréquentations sur certaines lignes familières, fut-ce de l'espace «vécu ou subit», ou encore le fait d'aller au même hyper-marché ou aux mêmes bureaux, ne crée pas nécessairement des solidarités territoriales, ni en termes de développement possible (théorie des pôles de développement!) ni en termes d'affectivité. Force est donc de constater à l'analyse qu'aussi bien au niveau de l'espace social objectif, considéré comme cadre spatial dans lequel évolue un individu ou groupe, qu'au niveau de l'espace social tel qu'il est subjectivement perçu et connu par les sujets et les groupes «qui le reconstruisent mentalement pour eux-mêmes, dans des espaces sociaux subjectifs», la possibilité de saisir concrètement la notion d'espace social, et donc de la géographiser, s'avère de plus en plus difficile. Et pourtant la tentative au moins s'impose, dans la mesure où, justement, en fonction même de ces différents modes de perception et de connaissance, les sujets et les groupes modifient leurs comportements, et tentent, ou du moins contribuent, à modifier de nouveau le cadre dans lequel ils vivent et les structures de l'espace social des représentations géographiques «normales» (DEMATTEIS, 1975)

Encore qu'un tel énoncé, souvent mobilisé pour justifier les tâches de la géographie de la perception en la liant à celle des comportements, soit particulièrement dangereux pour qui oublie une autre dimension de toute dynamique des espaces sociaux. Dans le cadre de l'appropriation et de l'aménagement du territoire, il existe en effet, à côté des pratiques des usagers, celles des décideurs/aménageurs agissant pour entretenir un élitisme politique et professionnel leur permettant le contrôle social des premiers, maintenus le plus souvent dans l'ignorance de leur réelle capacité d'action (CORDEY, 1985), l'Etat étant le garant politique d'un tel système. Manière comme une autre de rappeler ici la nécessité dans laquelle nous sommes de combiner dans nos études les multiples niveaux d'appréhension de la réalité sociale, combiner l'information «structurante saisie à petite échelle, à l'information factuelle saisie, elle, à grande échelle (RACINE, RAFFESTIN et RUFFY, 1980), la première rapportant des formes organisationnelles du niveau décideurs/aménageurs, la seconde, qui doit s'associer à la première, tendant à privilégier les pratiques des usagers. C'est ce qu'a réalisé, avec beaucoup de bonheur, un Philippe CORDEY dans son essai d'écologie humaine consacré à Genève sous le titre Appropriation et aménagement du territoire (CORDEY, 1984), en montrant en particulier comment la rénovation urbaine a conditionné pour les usager, le passage d'une territorialité à une autre, un nouveau système d'échanges et de communication débouchant, pour les usagers concernés, sur une série de coûts socio-économiques dont le géographe d'aujourd'hui ne peut plus se permettre d'évacuer l'étude. Celle-ci, recensant les malaises relationnels prétéritant toute possibilité de s'identifier à son environnement quotidien, risque bien d'identifier des processus de production sociale remettant radicalement en cause la notion même d'espace social. Celle-ci connote encore une certaine dose au moins d'enracinement dans un lieu, de proximité sociale et d'intégration locale, mais ne s'évanouit-elle pas quand le système relationnel devient de plus en plus asocial? Les réactions négatives, si communes aujourd'hui en Suisse, face à tous les projets de modification de l'espace intra-urbain proposés à la population en témoignent à contrario, comme aussi les signes très nets de revalorisation des patrimoines et autres vieilles pierres de nos centre-villes, voire même certains résultats statistiques (contre-urbanisation des dernières années). Et que dire de tant d'autres «réactions» saisies au niveau micro-social, dans lesquelles on découvre les indices au moins d'un retour du pendule face à la domination centralisante et centralisatrice d'une techno-bureaucratie dominante au sein de notre "société programmée», valorisations se situant à l'opposé de celles ayant donné (ou accompagné la) naissance à cette articulation centre-périphérique, si manifestement «déterritorialisante»? (BASSAND et HAINARD, 1985).

En définitive, pourra-t-on encore longtemps parler d'espace vécu, d'identité et de culture locale ou régionale, quand notre territorialité, de «régionalisée» qu'elle était; constituée par l'ensemble des relations à l'altérité vécues à travers le travail, l'habitat et certaines cristallisations traditionnelles, est devenue une territorialité «temporalisée» liée à un système de relations qui dépend en fait de la seule variation de la quantité d'information dans un territoire donné, information consommée, l'identité étant conditionnée par les modèles culturels dominants, nos relations étant bien moins conditionnées par ce territoire que par l'information qui y est diffusée, la base même de notre territorialité étant à chercher désormais dans des territoires abstraits (RACINE et RAFFESTIN, 1983; RAFFESTIN, 1980). 


\section{En guise de conclusion provisoire: la reconnaissance de l'intentionnalité}

Nous reconnaîtrons en ce qui nous concerne que si la terre est bien, phénoménologiquement, et comme le voulait Eric DARDEL (1952) au départ d'un merveilleux ouvrage resté plus de trente ans ignoré de ses pairs français, une "écriture à déchiffrer», les espaces sociaux attendent leur lecteur, et si la géographie est d'abord «conscience géographique» d'un rapport intentionnel à la terre, faire une géographie des espaces sociaux revient à découvrir, ou plutôt à retrouver l'intention en jeu dans ce rapport à la terre... à travers - et, c'est je crois la grande leçon que nous devons tirer de la pensée phénoménologique - le rapport intentionnel à la terre du géographe lui-même. Ayant renoncé à réduire le monde à un mé-canisme, ayant reconnu l'intention humaine en recherchant le sens et les significations (RACINE, 1986b), nous créons l'espace théorique pour les espaces sociaux. Nous «lirons» certes l'espace, mais celui-ci n'est pas ce bavard vers lequel nous n'avons qu'à tendre l'oreille (RACINE, 1986c). L'espace, tant celui du scientifique que celui qu'il étudie, est une lecture, une interprétation d'un texte dont nous sommes les auteurs. Tant et si bien qu'aujourd'hui, à la différence de nos prédécesseurs, nous reconnaîtrons que si l'espace du géographe est bien un produit social, progressivement construit par les sociétés à leur image dans l'historicité de leurs pratiques - c'est là nous semble-t-il un point acquis - cet espace prend une figure marquée de différentes qualités par l'intermédiaire des sujets qui l'appréhendent, le marquent et l'informent, organisant ses différentes déterminations en ordres signifiants. C'est assez dire que cet espace n'est pas «objectif», fonction univoque de choses, mais produit à la fois de la lecture d'un individu et de la détermination qu'exerce sur lui la société, l'histoire, la langue, que sais-je encore... Cherchant un sens, le géographe, par là même, comme le sémioticien, le suscite. L'espace n'est plus cette opacité qui attendrait la perspicacité du géographe scientifique pour apparaître dans sa muette évidence. Il est le support des finalités de chacun: en lui se superposent et s'affrontent les finalités des particuliers comme des collectivités: des lectures simultanées ou successives montrent à l'évidence la multiplicité des enjeux qu'il représente. «En lui» n'est d'ailleurs pas l'expression qui convient. L'espace n'est pas un receptacle neutre que viendraient recouvrir des lectures, sorte de trame neutre a priori, table rase dont on pourrait envisager de retrouver la pureté originelle. En fait, l'idée même d'espace, comme de vouloir en retrouver la pureté ou les principes d'organisation, sont déjà une lecture, un rapport au monde, comme le sont toutes nos études examinant et questionnant les morphologies stratifiées socialement et géographiquement et qui relèvent d'un modèle culturel précis, le nôtre, celui d'une géographie qui entre autres, a évacué ses images - et les images - mais aussi toute l'affectivité qui imprégnaient, les unes les livres qui nous ont fait rêver des paysages et des hommes, l'autre le rapport d'empathie qui animait le plus souvent la saisie qualitative, historico-littéraire et verbo-conceptuelle de la géographie classique.

Il ne faut donc pas envisager la géographie, non plus que toute autre science (humaine), comme la tentation de retrouver un original dont on ne connaîtrait spontanément que la copie. L'espace devient en fait le grand livre des intentionnalités, que l'intentionnalité du «lecteur», du géographe, va tenter d'interprêter, en y mettant beaucoup de lui-même, à travers les signes qui seront comme les indices dans un jeu de pistes qui est désormais le quotidien de sa discipline.

A l'évidence, cette recherche des signes pertinents sur lesquels et à travers lesquels construire une représentation - car c'est toujours de cela qu'il s'est agit et qu'il s'agit en fait - des espaces sociaux, s'est inscrite et peut s'inscrire dans des problématiques fort différentes, qui tout en se contredisant parfois, en se rejettant réciproquement l'injure «réductionniste», nous paraissent moins s'exclure que se compléter, dans la mesure où elles se sont surtout enrichies les unes les autres avec le temps pour en arriver aujourd'hui à mieux «coller» au réel social, tel que vécu par les hommes se faisant et faisant leurs espaces à leur image en se faisant eux-mêmes, «un nouveau paradigme (n'étant) parfois que l'aboutissement de nécessaires réajustements» (AURIAC, 1986).

\section{Bilbiographie}

AURIAC, F. (1986): Du spatial et du social de la géographie aujourd 'hui. In: L'Espace: jeux et enjeux (sous la direction de F. Auriac et R. Brunet), La Nouvelle Encyclopédie, Paris (sous presse).

BAILLY, A. S. (1984): La géographie des représentations: espaces perçus et espaces vécus. In: Les concepts de la géographie humaine (A. Bailly ed.) Paris, 133-138.

BAREL, Y. (1986): Le social et ses territoires. In: L'Espace: jeux et enjeux, op. cit. infra.

BASSAND, M. et HAINARD, F. (1985): Dynamique socioculturelle régionale. Lausanne.

BRUNET, R. (1986): L'Espace, règles du jeu. In: L'Espace: jeux et enjeux, op. cit. infra.

CAMPICHE, R. et ZIMMERMANN, E. (1975): La vie sociale aux Eaux-Vives, I. E. S. Lausanne, Série spéciale d'Etudes et Rapports, Changement social et communauté, no 5. 
CLAVAL, P. (1973): Principes de géographie sociale, Paris. CORDEY, Ph. (1984): Appropriation et aménagement du territoire, Genève, essai d'écologie urbaine, Berne.

CORDEY, Ph. (1985): Pour une géographie écologique: interprétation d'une mise en jeu des acteurs à travers l'analyse des coûts sociaux de la rénovation urbaine. In: Cahiers de Géographie du Québec, vol. 29, no 78, 405-413.

DARDEL, E. (1952): L'Homme et la Terre, Nature de la réalité géographique, Paris.

DEMATTEIS, G. (1985): La metafore della terra, la geografia tra mito e scienza, Milano.

DEMATTEIS, G. (1985): Dans la tête de Janus, réflexions sur le côté poétique de la géographie. In: L'Imagination géographique (J. B. Racine et C. Raffestin ed., Géotopiques 1985 Genève-Lausanne, 109-125.

FOSSAERT, R. (1977): La société, Tome 2: Les structures économiques, Paris.

FREMONT, A. (1984): Esquisse pour une problématique de la géographie sociale. In: Sens et non-sens de l'espace, Collectif français de Géographie urbaine et sociale, Paris, 37-41.

FREMONT, A., CHEVALIER, J., HERIN, R., et RENARD, J. (1984): Géographie sociale, Paris.

GEORGE, P. (1978): Les populations actives, Introduction à une géographie du travail, Paris.

HERIN, R. (1986): Commentaires sur la géographie sociale, Débats de L'Espace géographique (sous presse).

LALIVE D'EPINAY, C., CHRISTE, E., BASSAND, M. (1978): Structure sociale et système des loisirs. In: Rev. suisse de sociologie, N. 3, 139-177.

MENETREY, A. C. (1982): La vie... vite, Lausanne bouge 1980-1981: une chronique, Lausanne.

POCOCK, D. C. (1984): La géographie humaniste. In: Les concepts de la géographie humaine, op. cit. infra, 139-142.

RACINE, J. B. (1985): La géographie cognitive à la recherche d'une épistémologie de son programme scientifique et critique. In: Les représentations en actes. Actes de Colloque de Les chevaines, Grenoble, Institut de Géographie Alpine, p. 329-348.
RACINE, J. B. (1985): De l'être et du phénomène dans la pratique de la géographie, récurrences épistémologiques à la lecture d'Eric Dardel. In Géorythmes, no 4 (sous presse).

RACINE, J. B. (1985b): Qualità della vita, benessere e mutamento sociale: verso una nuova geografia degli spazi vissuti e dei rapporti dell'uomo con il territorio. In: Esistere e abitare, Prospettive umanistiche nella geografia francofona, Milano, 199-246.

RACINE, J.B. (1986c): La terra, scritura du decifrare? In: L'uomo e la terra: Natura della realta geografica, Milano (sous presse).

RACINE, J. B., RAFFESTIN, C. et RUFFY, V. (1980): Echelle et action, contribution à une interprétation du mécanisme de l'échelle dans la pratique de la géographie. In: Geographica Helvetica, vol. 35, no 5, 87-94.

RACINE, J. B. et RAFFESTIN, C. (1983): L'espace et la société dans la géographie sociale francophone: pour une approche critique du quotidien. In: Espace et localisation, La redécouverte de l'espace dans la pensée scientifique de langue française, Paris, 304-330.

RACINE, J. B. et REYMOND, H. (1983): L'analyse quantitative en géographie, Paris.

RAFFESTIN, C. (1980): Pour une géographie du pouvoir, $\mathrm{Pa}-$ ris.

RAFFESTIN, C. et BRESSO, M. (1979): Travail, Espace, Pouvoir, Lausanne.

SCHULER, M. et NEF, R. (1983): Räumliche Typologien des Zentren-Peripherien-Musters. In: Rapports des travaux no 35, Berne.

SCHULER, M. et al. (1985): Atlas structurel de la Suisse, Zurich.

WALTER-BUSCH, E. (1980): Schweizer Regionenspiegel, Aarau.

WALTER-BUSCH, E. (1983): Subjective and Objective Indicators of Regional Quality of Life in Switzerland. In: Social Indictors Research, vol. 12, no 3, 337-391.

ZORN Fritz (1979): Mars, Paris, (trad. de l'allemend). 